\title{
Acetato de medroxiprogesterona en la enfermedad fibroquística de la mama en adolescentes
}

\author{
Dra. María Inés Sims D. ${ }^{1}$; Dra. Mónica Muñoz ${ }^{2}{ }^{2}$; Dr. Benita González M. ${ }^{3}$ \\ Cystic breast disease in adolescent girls. Treatment with medroxyprogesterone
}

Eighteen adolescent girls with moderate and severe fibrocystic disease of breast, whose mean age was 15.3 years (range 13 to 20 years) were treated along 3 menstrual cycles with daily oral doses of medroxyprogesterone for 10 days from day 14 of each period. Disappearance or marked reduction of breast pain and nodules occurred in 89 and $78 \%$ of the patients respectively. No undesirable effects were obsurved.

(Key words: fibrocystic disease of breast, breast dysplasia, medroxyprogesterone.)

La enfermedad fibroquística de la mama (EFQ) puede recurrir en el período de la adolescencia y se caracteriza por dolor espontáneo o provocado de la glándula, junto con nódulos localizados o diseminados en una o ambas mamas $^{1,5}$.

En su patogenia intervienen varios factores, pero parece que el componente hormonal juega un rol importante, sea como elemento desencadenante o concomitante. La relación estrógenoprogesterona sería de gran importancia para mantener en la glándula mamaria los componentes epitelial y estromatoso en perfecto equilibrio. El incremento de estrógeno por déficit de progesterona podría aumentar la proliferación de los conductillos y precipitar la aparición de $\mathrm{EFQ}^{3}$.

Entre los diversos tratamientos empleados en la EFQ de la mujer adulta, el acetato de medroxiprogesterona (AMOP) ha mostrado buenos resultados con porcentajes de respuesta cercanos a $90 \%{ }^{1,4,6}$. El AMOP es un progestágeno sintéti$\infty$, cuya estructura química es muy cercana a la molécula natural y corresponde a la 6-metit17 -acetoxyprogesterona. Su acción principal se manifiesta a nivel progestacional, con intenso efecto antiestrogenico ${ }^{4}$.

El propósito de este trabajo es mostrar los efectos terapéuticos y colaterales del AMOP en la enfermedad fibroquística de la adolescente,

1. Jefe Unidad Ginecología Infantil y Adolescencia. Hospital L. Calyo Mackenna.

2. Unidad de Ginecología Infantil y Adolescencia. Hospital L. Calvo Mackenna.

3. Unidad de lnmunología. Hospital L. Calvo Mackenna. sobre los que existe escasa información (y se carece de investigaciones sistemáticas) en este grupo etario en la literatura nacional y extranjera.

\section{MATERIAL Y METODO}

Sc seleccionaron 18 adolescentes portadoras de EFQ que consultaron en el Servicio de Ginecolog ía Infantil y Adolescencia del Hospital Luis Calvo Mackenna durante el segundo semestre de 1986, cuyas edades fluctuaron entre 13 y 20 , mediana 15 , años. En su selección se tomaron en consideración el cuadro clínico y las con. centraciones plasmáticas de progesterona previas al tratamiento. Las manifestaciones clínicas más importantes para el diagnóstico de $\mathrm{EFQ}$ fueron dolor mamario y nódulos palpables. El dolor fue calificado en una escala arbitraria en: leve (molestias escasas que no se presentaban en todos los ciclos); moderado (presente en todos los ciclos durante el período premenstrual, requiriendo consumo esporádico de analgésicos) y severo (excede el período premenstrual, consumo trecuente de analgésicos $y$ afecta la actividad diaria). En el examen clínion del nódulo mamario se consignó la presencia de micronódulos ( $\leqslant$ de $3 \mathrm{~mm}$ ), la mayoria de las veces numerosos, que tendían a confluir en el tejido glandular normal; o macronódulo ( $\geqslant$ de $4 \mathrm{~mm}$ ), con o sin derrame del pezón (tclorrea).

Solamente ingresaron al estudio enfermas cuyo periodo mínimo de observación fue de 3 meses de enfermedtad, tuviesen al menos 1 macronódulo o un número de micronódulos no infetior a cin $\infty$ y concentraciones plasmáticas đe progesterona inferiores a $10 \mathrm{ng} / \mathrm{ml}$.

La medición de progesterona se realizó el dia $21 \mathrm{del}$ ciclo, mediante técnica de radioinmunoensayo, RIA.

Se excluyen del estudio adolescentes que presentaran otras afecciones concomitantes o estuvieran recibiendo tratamiento anticonceptivo.

El tratamiento se realizó con acetato de medroxiprogesterona (Fariutar $\left.{ }^{(}\right)$) en dosis de $10 \mathrm{mg}$ diarios, durante 10 días a partir del día 14 de cada ciclo. Este es- 
quema se mantuvo durante 3 meses consecutivos. Se controlaron las enfermas cada 30 djas, registrando los cambios en la intensidad del dolor, la tumoración mamariz y los posibles efectos colaterales sobre el peso corporal, la presión arterial, y la aparición de cefalea, molestias digestivas o ambas.

\section{RESULTADOS}

La edad de las pacientes varió entre 13 y 20 aก̃os ( $\overline{\mathrm{X}} 15,3$ años).

Once enfermas $(61,1 \%)$ tenían dolor. La nodulación fue predominantemente mixta en igual proporción de las pacientes (tabla 1).

El nivel de progesterona en el periodo ovulatorio es $2,5 \mathrm{ng} / \mathrm{mL}$; en las 18 pacientes la mediana fue $1,3 \mathrm{ng} / \mathrm{mL}$.

La frecuencia de la mastalgia disminuyó a través de los ciclos desde $61,1 \%$ al ingresar a sólo $11,1 \%$ en el $3^{\text {er. }}$ ciclo. La frecuencia de nódulos mamarios se redujo de 100 a $22 \%$ al completar
Tabla 1

Manifestaciones clínicas de ingreso, 18 adolescentes con displasia quística de la mama

\begin{tabular}{lrr}
\hline Dolor & 7 & \\
Ausente & 2 & 38,9 \\
Leve & 8 & 11,1 \\
Moderado & 1 & 44,4 \\
Severo & & 5,6 \\
& & \\
- Nodulaciones & 2 & 11,1 \\
Micronódulo & 5 & 27,8 \\
Macronódulo & 11 & 61,1 \\
Mixto & 7 & 38,9 \\
\hline
\end{tabular}

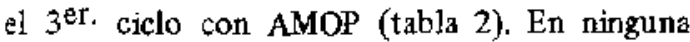
paciente se detectó derrame del pezón y no se observaron efectos colaterales atribuibles a AMOP durante los 3 ciclos del tratamiento.

Tabla 2

Evolución manifestaciones clínicas en pacientes con EFQ tratadas con acetato de medroxiprogesterona $\mathrm{N}^{\mathrm{O}}=18$

\begin{tabular}{|c|c|c|c|c|c|c|c|c|}
\hline \multirow[b]{2}{*}{ Parámetro } & \multicolumn{2}{|c|}{ Ingreso } & \multicolumn{2}{|c|}{1 er. ciclo } & \multicolumn{2}{|c|}{$2^{\circ}$ ciclo } & \multicolumn{2}{|c|}{$3^{\text {er. ciclo }}$} \\
\hline & నুo & $\%$ & No & $\%$ & No & $\%$ & No & $\%$ \\
\hline - Dolor & 11 & 61,1 & 9 & 50,0 & 8 & 44,4 & 2 & 11,1 \\
\hline - Nodulación & 18 & 100,0 & 18 & 100,0 & 7 & 38,9 & 4 & 22,2 \\
\hline
\end{tabular}

\section{DISCUSION}

La frecuencia de EFQ en la mujer adulta que consulta por patología mamaria es cercana a $40 \%$ y oscila entre 20 y $70 \%$ en la población general.

A pesar de los cambios regresivos que experimenta la mama durante la menopausia, se describen en algunos casos de EFQ aun hasta los 70 años ${ }^{2,5}$.

En una serie de 520 casos publicados en Chile, la frequencia de EFQ entre los 10 y 20 años de edad fue de $5,96 \%{ }^{5}$.

En nuestra unidad, en el afío 1986, la patología mamaria alcanzó a $20,5 \%$ del total de las consultas y dentro de aquélla la EFQ fue la más frecuente.

El dolor mamario, espontáneo o provocado, fue generalmente difuso $y$ bilateral en este grupo de adolescentes. La sensación de turgencia y ple- nitud mamaria, con nódulos chicos confluentes, medianos a grandes, lo pudimos comprobar en la mayoría de ellas.

Por cuanto la lesión compromete el epitelio de los conductos, puede aparecer derrame del pezón, preferentemente seroso, pero cuando se asocia a lesiones papilares puede ser sanguj. nolento ${ }^{5}$. Este signo no lo observamos en el gnupo estudiado.

Numerosas publicaciones demuestran déficit lúteo, del tipo fase lútea corta o inadecuada $a^{6}, o$ concentraciones normales de estrógenos con las de progesterona significativamente inferiores. $\mathrm{Se}$ considera que un descenso leve de la progesterona puede ser suficiente para originar síntomas mamarios, y que cuando ésta es menor que 10 $\mathrm{ng} / \mathrm{mL}$, corresponde a insuficiencia lútea, que requiere terapia con progestágenos. Esta última situación se identificó en la mayoria de nuestras 
pacientes $(94,4 \%)$. Por 10 antes expuesto, valdria la pena considerar la teoría del desbalance hormonal.

Se describe en la mujer adulta la utilización de dosis altas de AMOP, con la consecuente alteración transitoria de los ciclos menstruales, en el manejo de la $E F Q$, con respuesta hacia una regresión total o marçada del nódulo mamario en más de $70 \%$ de los casos y del dolor mamario en más de $90 \%{ }^{6}$.

Nosotros utilizannos dosis bajas de AMOP, con resuitado similar en cuanto a la regresión de los síntomas y signos. No observamos alteración del flujo rojo u otros efectos colaterales.

En sintesis, la EFQ constituye parte importante de la patología mamaria de la adolescente. Si bien hay diferencias, desde el punto de vista clínico y hormonal con lo que ocurre en la mujer adulta, no es posible descartar un origen similar en ambos periodos de la vida. Se dispone de terapia eficaz, el AMOP, sin efectos colaterales y con resultados clínicos favorables. El efecto clínico beneficioso del AMOP puede deberse a un significativo descenso en los niveles plasmáticos de estrógenos, como sucede en la mujer adulta que es tratada con esta progesterona sintética.

\section{RESUMEN}

Estudio prospectivo en el que se examinan 18 adolescentes con mastopatía fibroquistica moderada y severa, que se trataron durante 3 ciclos con acetato de medroxiprogestorona (AMP). Los resultados sugieren que el AMP es un agente farmacológico efectivo en este grupo de pacientes sintomáticas, desapareciendo la nodulación en el 78\% y la mastalgia en el $89 \%$. No se observaron efectos colaterales. Es importante el control y seguimiento.

\section{REFERENCIAS}

1. Peralta O., Campodónico I.: Ensayo terapéutico con medroxiprogesterona, acetato, en la mastopatía fibroquística severa. Rev Chil Obst Ginecol $1983 ; 48,146-155$.

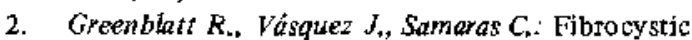
Breast disease. Postgrad 1982; 71, 159-168.

3. Nezhat C.: Fibrocystic disease of the Breast. Clin Obstet Gynecol 1982; 25: 365-371.

4. International Symposium of medroxyprogesterone Acetato (MPA). Book of Symposium. Geneva (Switzerland), February, 1982.

5. Pabst I.: Mastopatía fibroquistica. Arch Soc Cir Chile 1970; 17: 69-76.

6. Peralto O., Campodónico I.: Mastopatía fibroquística. Modificaciones clínicas y homonales con la administración continua de acetato de madroxiprogesterona, Rev Chil Obsiet Ginecol 1986; $6: 470-477$.

7. Manvais-Jarvis $P_{1}$, Sitruk-Ware $R$, Kutten $F_{.}$: Beningn Breast Cancer. Advances in Research and treatment. W.C. Mc Quire Ed. 3.

8. Korenman S.U.: The endocrinology of breast cancer. Cancer 1980;47:874. 